\title{
Project-Based Learning Among Engineering Students During Short-Form Hackathon Events
}

\section{Mr. Paul Alexander Horton, Arizona State University}

Paul Horton is an undergraduate and graduate student at Arizona State University studying Software Engineering (MS, BS) and Applied Physics (BS). He is currently working on research projects at NASA Jet Propulsion Lab on optical communications and Mars surface transient classification using machine learning. He hopes to continue his education into Astrophysics and use his software background to study the universe.

\section{Dr. Shawn S. Jordan, Arizona State University, Polytechnic campus}

SHAWN JORDAN, Ph.D. is an Associate Professor of engineering in the Ira A. Fulton Schools of Engineering at Arizona State University. He teaches context-centered electrical engineering and embedded systems design courses, and studies the use of context in both K-12 and undergraduate engineering design education. He received his Ph.D. in Engineering Education (2010) and M.S./B.S. in Electrical and Computer Engineering from Purdue University. Dr. Jordan is PI on several NSF-funded projects related to design, including an NSF Early CAREER Award entitled "CAREER: Engineering Design Across Navajo Culture, Community, and Society" and "Might Young Makers be the Engineers of the Future?," and is a Co-PI on the NSF Revolutionizing Engineering Departments grant "Additive Innovation: An Educational Ecosystem of Making and Risk Taking." He was named one of ASEE PRISM's "20 Faculty Under 40" in 2014, and received a Presidential Early Career Award for Scientists and Engineers from President Obama in 2017.

\section{Steven Weiner, Arizona State University, Polytechnic campus}

Steven Weiner is a PhD student in Human and Social Dimensions of Science and Technology at the School for the Future of Innovation in Society at Arizona State University. His interests include STEM education reform, innovative learning frameworks, and the future of schooling. His previous research focused on how young adults develop identities centered on the Maker Movement and his dissertation will explore the effect Maker-based initiatives, such as the establishment of school makerspaces, are having on the culture of formal educational institutions. Before starting his doctoral studies, Mr. Weiner served as the founding Program Director for CREATE at Arizona Science Center, a hybrid educational makerspace/ community learning center. He has previous experience as a physics and math instructor at the middle school and high school levels.

\section{Dr. Micah Lande, Arizona State University}

Micah Lande, Ph.D. is an Assistant Professor in the Engineering and Manufacturing Engineering programs and Tooker Professor at the Polytechnic School in the Ira A. Fulton Schools of Engineering at Arizona State University. He teaches human-centered engineering design, design thinking, and design innovation project courses. Dr. Lande researches how technical and non-technical people learn and apply design thinking and making processes to their work. He is interested in the intersection of designerly epistemic identities and vocational pathways. Dr. Lande received his B.S in Engineering (Product Design), M.A. in Education (Learning, Design and Technology) and Ph.D. in Mechanical Engineering (Design Education) from Stanford University. 


\title{
Project-Based Learning Among Engineering Students During Short-Form Hackathon Events
}

\begin{abstract}
Collaborative hackathons give undergraduate engineering students an opportunity to work in small teams to solve technical design challenges in a severely constrained time period. These events approximate project-based learning environments by giving students the opportunity to learn new technical skills through projects of their choosing. Project-based learning classes often face problems with class time usage and students having difficulties understanding learning objectives. Hackathons may provide insight on how to improve the implementation of projectbased learning curricula while increasing student interest and engagement.
\end{abstract}

\section{Introduction}

The emergence of project-based learning (PBL) within the engineering education realm has provided opportunities to apply their technical knowledge to engineering projects. ${ }^{1}$ The aspects of this approach usually have teams of students working to complete an authentic design challenge. The students learn in these classes through facing challenges in a project of their choosing that requires them to acquire new skills. Students can learn these skills through traditional instruction from an educator or through self-learning. The benefits of this type of class come from the exposure that students get to real world design approaches. Unfortunately, this course structure requires a large portion of class time to non-technical topics and burdens the professor with developing project prompts which can take up much of their course planning time. In instances where this type of course is not executed properly, students are overcome with the ambiguity of the project and are unable to attach themselves to the project and ultimately learn. ${ }^{1}$

Hackathons are an interesting example to look at as they are similar to PBL in many aspects. A typical hackathon approach to PBL follows an unconventional route when compared to the classroom setting. Their primary structure is a short-form programming sprint that takes place over 24 to 48 hours in which teams of participants focus on making a working product for demoing at a showcase. In this environment, demoing is a mark of success as it demonstrates that the team has created something they are willing to show to the public. While the structure and timeframe differ significantly, the hackathon environment encourages the same type of innovation one would expect from a PBL course. Hackathons, however, have not previously been thought of as an environment for PBL in the same way that classroom courses have. Given that hackathons are becoming increasingly integral to the success of computer science students, it is useful to consider how, and to what extent, elements of PBL are manifested in hackathons. ${ }^{4}$ The purpose of this research study is to use the Buck Institute of Education's (BIE) Gold Standard PBL framework to analyze hackathon environments for the presence of key projectbased learning characteristics. ${ }^{2}$ This was accomplished through a qualitative study utilizing both observation and interview protocols of selected teams at Major League Hacking events.

\section{Background Information and Literature \\ A. Hackathon Background}

Hackathons are a relatively new phenomenon in terms of research and as such there are very few studies within the engineering education literature. Major League Hacking, a B-corp organization that partners with collegiate hackathons to provide organizers with resources and 
advice, describes hackathons as an "invention marathon," where people interested in technology can "learn, build and share," their creations with others. ${ }^{3}$ These events have attracted 65,000 student attendees at 200 unique hackathons at different universities in 16 countries. ${ }^{3}$ It is important to note the distinction between the security definition of hacking and a hackathon. Hackathons focus on hacking where "hack is used in the sense exploratory and investigate programming." ${ }^{4}$ Hackathons have nothing to do with technical security. Hackathons probably originated from Local Access Network (LAN) parties, in-person video game sessions, being mixed into programming sessions. The characteristics of a LAN party include little to no sleep, highly-caffeinated drinks, and technologically inclined individuals having fun doing something they love. ${ }^{4}$ These elements are all found in hackathons where likeminded individuals meet up to create some innovation under similar, pizza-fueled conditions.

The typical hackathon is structured around teams of four hackers (hackathon participants) having 24 to 48 hours to create some sort of "demo-able" computer science-based project. The projects are selected by the teams with the knowledge of specific prize categories. These prizes are typically geared towards socially-relevant coding but can also be specific to the sponsor providing them (e.g. Intel giving a prize for best use of their hardware). Teams of hackers are then formed and they spend the duration of the hackathon attending tech talks (short workshops to introduce new techniques), participating in fun tech related activities (like recreating HTML websites without syntax highlighting), and, of course, working on an innovative project. The majority of these activities help the participants learn new skills without pressuring them into learning specific skills. This experience also helps hackathon hackers connect with industry professionals as "hackathons have elements of a job fair allowing industry sponsors the chance to watch specific students in action," [6]. Individuals demonstrate their potential value to companies through hackathons by showcasing their ability to tackle projects and new skills in high pressure environments. At the end of the development cycle, hackers demo their projects to the public in an exhibition hall showcase.

\section{B. Project Based Learning Background}

Project-Based Learning is an approach to education that engages "students in [a] sustained collaborative focus on a specific project, often organized around a "driving question," framework for PBL that we utilized in this study is the BIE Gold Standard PBL. BIE has been chosen for this study as its PBL framework is most easily adaptable into a thematic analysis framework where the elements define the codes. BIE describes PBL as having three parts: Student Learning Goals, Essential Project Design Elements, and Project-Based Teaching Practices. For this study, we looked specifically at the Student Learning Goals and the Essential Project Design Elements to assess both the learning outcomes for hackathon participants as well as the structure of the hackathon itself. See Figure $1^{2}$. 


\section{Figure I: Buck Institute of Engineering Gold Standard Project-Based Learning Framework}

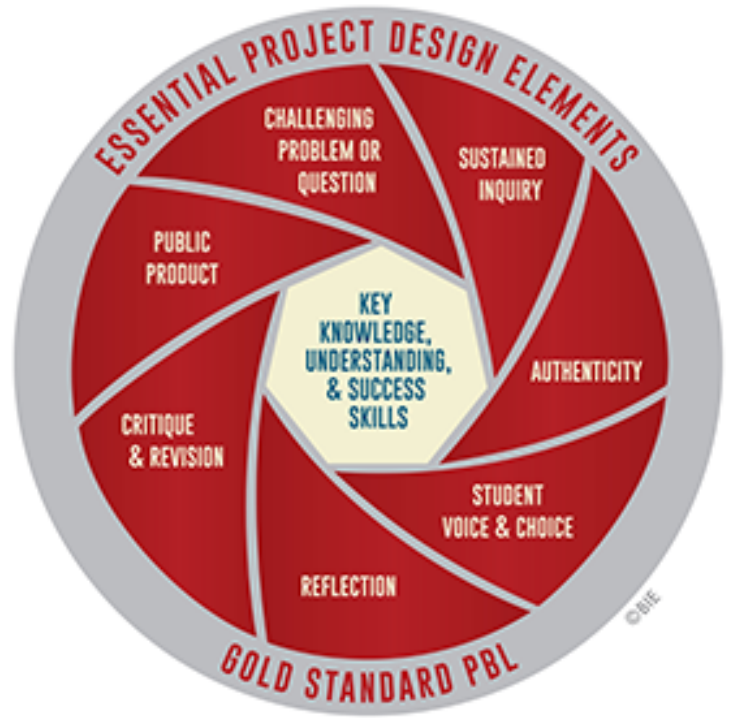

\section{Student Learning Goals}

Students in a PBL environment should be able to gain key knowledge and understanding of the desired concepts and content standards. This means that the students will not only learn the required material but "learn how to apply knowledge to the real world." Beyond learning knowledge, students will also learn important skills regarding their ability to work in teams and management of themselves and others. These skills are regarded as " 21 st Century Skills" and are vital to student's success as they enable students to thrive in the modern workplace. Projects in PBL should focus on "critical thinking/problem solving, collaboration, and self-management," in order to succeed in meeting these Student Learning Goals.

\section{Essential Project Design Elements}

These elements are a way to structure a PBL design project so that it best allows the environment to enable the BIE Student Learning Goals.

\section{a. Challenging Problem or Question}

At the heart of PBL is the project itself. A project should be investigative and allow students to engage the problem in an open-ended manner. This makes the learning more meaningful for students because they will have a "real need to know something, so they can use this knowledge to solve a problem or answer a question that matters to them." BIE recommends that projects should be like a thesis focus for an essay, open-ended and challenging without being intimidating or limiting creativity.

\section{b. Sustained Inquiry}

PBL directs students to investigate their solutions prior to starting their project. This goes beyond "looking something up," and focuses on in depth research to solve the problem. This means that when confronted with a problem, students should ask deeper questions and keep doing so until they find a satisfactory solution. For products, students should look to the users of their potential 
product and see what sorts of solutions they are in need of. This design process allows students to become more invested in the project and help them create a more viable solution.

c. Authenticity

The authenticity of a project is something that will help motivate the student's learning. A project can have an authentic context in which students directly solve problems faced by people outside the classroom. This can be structured like a real-world project in which students must come up with viable solutions to a real-world problem. This type or project can have a real impact on communities which will help students become more motivated to use their best effort to solve the problem. The project can also be authentic to the student by making it relevant to their own interests or issues in their lives. This can be done through more ambiguous problem statements that give students the freedom to explore possible projects.

d. Student Voice and Choice

Students in a PBL course should have a say in the structure of their project. This creates a sense of ownership among the students and helps them to work harder to accomplish their goals. This makes the project less like an exercise and more personal to them. Giving students control over aspects of the project allows them to be more creative when designing their solution. For advanced courses, students may even be the one to select the topic and nature of the project by developing their own project questions and defining project success in their own terms.

\section{e. Critique and Revision}

Students in a PBL environment should be able to critique other projects and revise their own project based on criticism. This will ensure that the projects they create are that of a higher quality. This is different from a typical classroom as it is not just the teacher providing feedback on the project but other individuals such as other students, experts, and peers. This gives the critique and revision of the project a real-world point of view and helps enforce the authenticity brought up earlier.

\section{f. Public Product}

The final element of PBL courses is the project presentation. This gives the project an authentic feel as students will be mindful of showcasing their project at the conclusion of course. "When students have to present or display their work to an audience beyond the classroom, the performance bar raises, since no one wants to look bad in public."2 This presentation anxiety helps to drive better projects and should act as a healthy motivator. This public focus also ensures that the finished project has a social dimension that goes beyond the viewpoint of the teacher. An added benefit to this is that is shows to the community what students are capable of which in turn brings support to PBL from community stakeholders. This could mean more interest in PBL courses or even opportunities for outstanding individuals from outside the classroom.

\section{Research Design A. Purpose}

This research study was guided by the following question: How do specific elements of projectbased learning present themselves in the activities and interactions of members of a hackathon team? The development of this question came about through the first author's personal 
observations during hackathons and PBL classes in Software Engineering. The author noticed that most of the work done in his PBL classes ended up being completed in the few days before its deadline. This was especially relevant in classes where a burndown chart was present which almost always looked like a cutout of a continental shelf. In contrast, hackathons still have the same last-minute finishing, this time with hours before the deadline instead of days, but hackers tend to have more realistic goals about what they would like to accomplish. To the author, the efficiency of these events was something of interest and the author wanted to know to what extent the elements of PBL presented themselves in the setting. The author was also curious about how hackers in a hackathon feel about the skills they learn and how it compares to the skills they acquire in the PBL setting.

\section{B. Methodology}

This study follows a deductive thematic analysis of semi-structured interviews to develop a codebook. The study initially looked at teamwork in the hackathons setting but moved towards understanding PBL. Because of this shift, the data was collected prior to the PBL framework being selected. Deductively, the research team began using the BIE Gold Standard PBL framework as a structured and well-scoped framework to use for analyzing the data ${ }^{2}$. Data was collected through interviews and observations. The observational protocol was semi-structured and primarily focused on obtaining a history of a team's hackathon participation. Researchers would sit with the team for the duration of the hackathon making notes relating to their progress and way of overcoming problems. Interviews were conducted to obtain stories of their view on the project and were analyzed using thematic analysis with the BIE Gold Standard PBL as a framework. The codebook for this has descriptions of each from BIE as well as computer science specific examples that may be seen in hackathon setting. See Table I and II. 
Table I: BIE PBL Essential Design Elements Codebook

\begin{tabular}{|c|c|c|}
\hline Code & Description & Computer Science Example \\
\hline $\begin{array}{l}\text { Challenging } \\
\text { Problem or } \\
\text { Question }\end{array}$ & $\begin{array}{l}\text { A project that allows student to } \\
\text { engage in an open-ended manner } \\
\text { that passively requires them to learn } \\
\text { new skills. }\end{array}$ & $\begin{array}{l}\text { A final project for an embedded systems } \\
\text { class being to create an innovative } \\
\text { Internet of Things product. }\end{array}$ \\
\hline $\begin{array}{l}\text { Sustained } \\
\text { Inquiry }\end{array}$ & $\begin{array}{l}\text { Students having to do in depth } \\
\text { research on their project in order to } \\
\text { properly accomplish it. }\end{array}$ & $\begin{array}{l}\text { Students having to get community } \\
\text { feedback on the functionality of their app } \\
\text { through surveys. }\end{array}$ \\
\hline Authenticity & $\begin{array}{l}\text { A project having a real-world impact } \\
\text { that creates a context beyond the } \\
\text { classroom. }\end{array}$ & $\begin{array}{l}\text { A class partnering with a local non-profit } \\
\text { to develop apps to help organizer their } \\
\text { volunteers }\end{array}$ \\
\hline $\begin{array}{l}\text { Student Voice } \\
\text { and Choice }\end{array}$ & $\begin{array}{l}\text { A project that allows students to } \\
\text { have obtain ownership by giving } \\
\text { them judgement on the solution they } \\
\text { wish to implement. }\end{array}$ & $\begin{array}{l}\text { A class in which students pitch app ideas } \\
\text { to their professor and develop them for } \\
\text { the final project. }\end{array}$ \\
\hline Reflection & $\begin{array}{l}\text { Having students informally and } \\
\text { formally reflect on what, how, and } \\
\text { why they're learning }\end{array}$ & $\begin{array}{l}\text { Having students writing blog posts on } \\
\text { their development }\end{array}$ \\
\hline $\begin{array}{l}\text { Critique and } \\
\text { Revision }\end{array}$ & $\begin{array}{l}\text { Students should give and receive } \\
\text { constructive feedback on their } \\
\text { project and others so that they can } \\
\text { make improvements }\end{array}$ & $\begin{array}{l}\text { Pairing project teams and having them } \\
\text { discuss their solutions with each other. }\end{array}$ \\
\hline $\begin{array}{l}\text { Public } \\
\text { Product }\end{array}$ & $\begin{array}{l}\text { Requiring the project to be presented } \\
\text { to the public as a means for driving } \\
\text { higher quality, presentable, work }\end{array}$ & $\begin{array}{l}\text { A showcase at the end of the semester } \\
\text { where students display their findings. }\end{array}$ \\
\hline
\end{tabular}

Table II: BIE PBL Student Learning Goals Codebook

\begin{tabular}{|l|l|l|}
\hline Code & Description & Computer Science Example \\
\hline $\begin{array}{l}\text { Key } \\
\text { Knowledge } \\
\text { Understanding }\end{array}$ & $\begin{array}{l}\text { Learning knowledge and applying it } \\
\text { to real world problems and the } \\
\text { creation of high-quality products }\end{array}$ & $\begin{array}{l}\text { A student learning about Node.js and } \\
\text { then using that knowledge to create their } \\
\text { own web app. }\end{array}$ \\
\hline $\begin{array}{l}\text { Key Success } \\
\text { Skills }\end{array}$ & $\begin{array}{l}\text { Developing " } 21^{\text {st }} \text { Century Skills" } \\
\text { such as critical thinking, } \\
\text { collaboration and self-management } \\
\text { for their future success. }\end{array}$ & $\begin{array}{l}\text { A student working with peers on a } \\
\text { project that requires them to divide up } \\
\text { work to accomplish a collective goal. }\end{array}$ \\
\hline
\end{tabular}




\section{Data Collection}

To collect data, the research team created two protocols to observe teams in a hackathon setting. The first protocol is an observational protocol that highlights interactions between team members throughout the hackathon development process. The second protocol is an interview protocol that highlights individual team member's thoughts on hackathons, their project, their team, and their role in these areas. Once these protocols were developed we obtained IRB approval and began collecting data at national hackathons.

\section{Observational Protocol}

The observational protocol was semi-structured so that the data collection team could take notes beyond those labeled in the framework. These notes were primarily about how the team's morale, who was working on what, and how their progress was coming on the actual project. The notes also included the interactions between team members and their working environment. The data collected from these observations serves as a way to fill in the gaps in the data from the interview protocol.

\section{Interview Protocol}

The interview protocol is a list of semi-structured questions divided into pre-and post-data collection segments. The pre-interview was conducted shortly after team formation. The post interview was conducted after hacking ended and before demoing. The questions were generated to get a better idea on how participants felt about hackathons, their project, their team, and their role in these areas. Below are some examples of questions asked to highlight each area.

\section{Participant Selection and Project Approval}

The study comprised of three teams studied at two Major League Hackathon (MLH) hackathons during the 2017 season. Prior to collecting data, the research project received IRB approval to collect data on willing students 18 years old or older. Once we obtained university approval, we then approached organizers at MLH sponsored hackathons to gain their approval to collect data at the event. At the hackathon, teams were selected if all members met the following criteria: college student, older than 18 , participating in the event as a team, and willing to be observed. The individual members are given pseudonyms to protect their identity as per the IRB protocol. An overview of the teams observed is described here, labeled by their project:

\section{a. iOS College Freshmen Helper (Team A)}

This was a newly formed team with individuals who were not familiar with each other. This team intended to create an iOS App that helped students find the best fit college for them as well as help them through the process of enrollment and entering the college. What was unique was that the team focused on helping foreign students by providing information on travel and the process of coming to America for university. The final product was nowhere near their initial expectations and ended up being a framework demo of what the initially wanted.

\section{b. Virtual Reality Typing Teaching (Team B)}

This was a newly formed team with individuals who were familiar with each other but had not worked together on a project before. This team intended to create a bot for Slack that would integrate with an Amazon Alexa to send and receive messages as well as summarize missed conversations. The team pivoted ideas about a fourth of the way through when they realized that 
they were unable to accomplish this and turned to Virtual Reality where they wanted to make

Guitar Hero game. Their final project turned out to be a typing game that they realized would be perfect for teaching typing in an innovative way.

\section{c. Non-Drone Drone Mine Detector (Team C)}

This was a newly formed team with individuals who were not familiar with each other. This team intended to pursue Intel's prize challenge of using their four-wheel drive robotic platform. Using the robot, they wanted to create a demonstration of a landmine detection drone. Unlike the other teams, this team had a hardware focus with one member even creating his own metal detector from various parts found at the hackathon. The final product had the land rover with the metal detector sending data to a map interface that shows where metal is detected using GPS. This team was also a bit smaller and less talkative making data collection slightly more difficult for them.

\section{Data Analysis}

The collected data was analyzed using the Dedoose qualitative analysis software. Dedoose allowed the research team to create a codebook and code the interviews using thematic analysis. For this study the codebook was generated directly from the BIE Gold Standard PBL framework and highlighted themes that directly correlated to specific elements. The Student Learning Goals were also implemented in this codebook. The material was coded primarily with two individuals looking for the specific elements in the BIE framework and a larger team of five members validating the code. The unstructured observational protocol was used to fill in any gaps between the interviews and the framework and provide insight on things that weren't explicitly stated in the interviews.

\section{Results}

\section{A. Challenging Problem or Question}

Within the hackathon setting, there is typically no specific problem or question. Hackathons usually have prizes that direct hackers to design projects within a specific category. The only explicit requirement for a hackathon project is that it must be demoed in order to be deemed successful. The project being defined by prize categories and demoing fits perfectly with the Challenging Problem or Question element as it gives hackers a focus without explicitly giving hackers a project.

\section{Team B}

This team started the hackathon by writing every prize on a whiteboard and brainstormed how they could create a project that would optimize their chances at winning something. They came up with an app for Slack that would use an Amazon Echo because qualified for State Farm's Best use of Audio Commands as well as Amazon's Best Alexa Skill prizes. Mark explained that their team initially "want[ed] to go out and win all the prizes," (Mark Post). Even when the team pivoted to their virtual reality project, they still tried to develop for the Amazon Echo so that they could apply for those prizes. The team also used their project as a learning opportunity as Mark explains that they "want[ed] to learn more about evolving VR, cause none of us have done that before," (Mark Post). 


\section{Team C}

This team wanted to create a solution that incorporated humanitarian engineering and decided to do mine detection with drones. At the start of the hackathon they went directly to the hardware station to see what they could get their hands on to start their project. Having obtained a Hercules land rover, the team worked to use Intel's hardware to complete their project. Jim explained that they were "creating metal detectors for drones, like to attach[ed] onto drones, but since there's not a drone here and we don't have that, we're doing it on the little rover that's provided by Intel," (Jim Pre).

\section{B. Sustained Inquiry}

The hackathon setting does not do much to promote sustained inquiry as it is driven by the "develop first, ask questions later," mindset due to the timeframe. Given more time, teams may research their project solution more in depth but, for the most part, hackers come to the table with projects that aren't well thought out. Some teams, however, have ideas prior to the hackathon and research them outside of the event.

\section{Team C}

One of the members of this team was very experienced with hackathons and indicated that he was interested in solving world problems with his projects. Devin explained that prior to the hackathon he researched a potential project: "So for the whole thing, last night I was like, "Oh, I need an idea for the hackathon." So I figured I could do some sort of humanitarian thing, and I decided on mine detection. So I just did research. How do people detect mines? What are they looking for? How are they looking for them? Just like answered the who, what, when, where, why. And then when you go to, and now it's coming up with solutions. It was really, what are people doing? How could you do it better? And what are they not doing? What can I do? What are they doing? It was just a lot of questions. A lot of research," (Devin Pre).

\section{Authenticity}

All of the projects in a hackathon setting have some level of authentic context to them as the focus on demoing is intended to steer teams to create something that the public would be interested in as a product or solution to a problem. While not explicitly authentic, teams give their project authenticity focusing on solutions that have an impact to them and the world.

\section{Team A}

The team created authenticity in their project by directing it towards something personal to them- getting into college. For many of the team members, entering college was an interesting process as they we're international and minority students. The authenticity of this project comes from their own personal experiences with the process and their desire to make a solution to a problem they personally faced. When describing the project, John, an international student, explained that "It's basically a guiding app for college freshman, especially international student," (John Post). He explained that the app would help with things like visas and flight information, problems he faced in the past. 


\section{Team B}

Initially, the authenticity of the project came from the fact that many of the members on the team had an Amazon Echo and used Slack regularly. While they didn't necessarily see their product as a solution to an important problem, the team was interested in the possibility of this solution being usable to a larger audience. Ben commented on the usefulness of the project, "you talk to Alexa... you're talking to your channel through the Alexa. But I don't know. That's like, not that useful, and it probably would just be more annoying than just typing. It's one of those things, like, you could use Siri or you could just pull out your phone and it'd probably be easier. So this is something we think we could add actual usefulness," (Ben Pre). Alex explains that the environment itself created an authentic context. "[When needing to learn something] you have to go find it yourself and, or talk to the sponsors and people who are willing to help. But that's really cool because this is a much more realistic situation in which we're presented with a project," (Alex Post).

\section{Team C}

The authenticity of this project comes from the humanitarian aspect of it. As stated before, the team was very interested in solving real world problems so they choose a project that would have a real world impact.

\section{Student Voice and Choice}

Much like the Challenging Problem or Question element, hackers have free reign over what they want their project to be. BIE says that for advanced students, the topics themselves can be selected by the students. Hackathons exemplify this as the hackers define what they are interested in accomplishing. This allows them to become more connected to the projects they wish to create.

\section{Team B}

The team had complete control over what they wanted to do in order be as competitive for prizes as possible. The team chose the prizes that interested them most and designed a project around those prizes. After their mindset switched from, "We want to go out and win all the prizes," to be, "We'd rather just want to learn more about evolving VR, cause none of us have done that before,"” the team was still expressing interest in having control over their project (Mark Post).

\section{E. Critique and Revision}

Throughout the hackathon, teams are encouraged to talk with other teams about their projects. This happens naturally as everyone tends to be working in an open area allowing for an abundance of cross pollination between teams. Mentors and industry experts are also available to talk with and many teams utilize these individuals to get feedback on their project. Some teams do tend to keep to themselves though and do not seek feedback from outside sources.

\section{Team B}

Throughout the process, the team had many friends come in to test out their project. This was more prevalent when the team switched to Virtual Reality as it was more readily sharable with others. The team also had long discussions with the Amazon employees on implementing Alexa skills and using Amazon Web Services. The team also did internal revisions when they realized that their initial project wasn't going to work properly. Alex explains their pivot "we weren't 
really sure if there was an end in sight as far as the authentication goes. So at that moment we had to kind of make a pivot because we didn't know if we worked three more hours on the authentication, it just would have been too much time taken away to build the actual project, which is what we all wanted to do. So at that point, we just decided to build something that we all wanted to learn about, which was making a virtual reality game," (Alex Post).

\section{F. Public Product}

At the end of the hackathon, teams are given the opportunity to demo their project in an exhibition hall format showcase. Teams are not required to demo but they are heavily encouraged to do so as showcasing is when the judges circulate and evaluate the projects. When showcasing, teams get "I Demoed" stickers specific to the hackathon they are attending. Hackers view these stickers as a badge of honor and a sign that their hackathon experience was successful.

\section{Team B}

The final product was in a state that did not have every feature they wished to implement but found itself in a working state. Mark explains that "every part was pretty much individually done, but the game and the server are separate and they're not functional together yet," (Mark Post). At the start of the hackathon, the Steven's goal was to create something worth showing. "Even if I don't fully finish something, I like to get a comfortable point where I can show it," (Steven Pre). The team did exceptionally well during the demoing and managed to move onto the mainstage to demo during the closing ceremony. From there the team won first place in the overall competition.

\section{G. Key Knowledge and Understanding}

Throughout the hackathon, teams are given opportunities to interact with mentors and industry experts to ask questions about areas they may not be familiar with. During the hackathon there are also "Tech Talks" that introduce new and useful skills to hackers who attend. Across the board, almost all hackers studied indicated that they came to the hackathon to learn new skills and apply them to a cool project.

\section{Team A}

The members of this team gained a lot of exposure to Swift as that was the primary development environment for the project. John explained that he, "did learn a lot, but I think the really important part for me is that I'm at the very, very beginning of Swift programming, so this is a good start, and I think I will use it in the future," (John Post). The team also went to many tech talks that they found beneficial. Pat explained that the whole hackathon had elements where "you might have a teammate that knows this new skill that you don't know, or no one may know and so you have to discover it yourself," (Pat Post).

\section{Team B}

The team initially was looking for a project that gave them the highest opportunity at winning prizes but eventually moved to a project they felt they'd get the most out of educationally. Mark explains that hackathon learning is less formal and emphasizes the welcoming atmosphere hackathons create when it comes to learning. "When you're learning it, when you're using it, you're not necessarily doing so in order to digest the information, you're doing so because you 
need it to work. So I don't know whether that's better or worse, but I also feel like it lets you prototype more rapidly and get experience with a lot of things. And part of it's nice because you're not necessarily worried about how well it works, or if it's perfect, you just want to use it to some degree. So I think that can help people just get an idea of how or what to do, and not necessarily what the perfect and right way to do things is. Because a lot of times in software, there isn't necessarily a perfect and right way," (Mark Post). Alex also discusses learning by stating, "I think university courses, you're kind of presented with information and not much application, whereas Hackathons it's very much the reverse where you need an application, and you don't have the information," (Alex Post). This "need an application" mindset is exactly how PBL hopes to promote learning.

\section{Team C}

Tyler expressed that this hackathon exposed him to new skills such as "How to build an RL circuit and a metal detector and also just on the coding side quite a bit just interfacing with different microprocessors and a lot of Linux and [Arduino] Nano, some new coding languages that I hadn't been exposed to I got to get a taste of," (Tyler Post). He also expressed that the skills he learned, "are going to stick with me a bit more because I can see their direct and immediate practical application as opposed to a lot of more theoretical stuff. With the adrenaline and comradery, this will probably stick with me more," (Tyler Post).

\section{H. Key Success Skills}

The hackathon environment requires that hackers communicate and work efficiently to complete their task in the allocated time. For newly formed teams this means that they must learn each other's work habits quickly and figure out ways to collaborate. For teams with familiar member this means enabling what is already known about each members' skills so that the project is completed on time. Teams also must make good use of the resources at their disposal such as mentors and hardware/software. Finally, teams must exercise their presentation skills when demonstrating their project to the public.

\section{Team A}

Pat had some great insight on how the hackathon environment helped encourage good management skills. He stated that there was a level of integrity to the team's participation that is uniquely found in hackathons. "You can watch what they're [your teammate] doing and also you're right there to help them. You're spending more amount of time with your teammates next to you than a traditional classroom assignment." Max also indicated that the hackathon setting helped encourage project management. "I think at a Hackathon it's presented in a way you want to build something that you want to build. I think it has a lot to do with motivation," (Max Post). Max explains that this motivation comes from the fact that "You are all in the same boat, and you guys are all wanting to build a product that you're all happy and proud to display," (Max Post).

\section{Team $B$}

Alex also had insight on the hackathon environment and its motivational aspects, "I think the motivation is definitely higher. I would say so because, I mean, there's more at stake here. It's not only the prizes, but it's also the social aspect, and it's really just fun staying up all night coding to have these sessions. It's four in the morning. You're tired, but your creativity is still flowing," 
(Alex Post). Prior to hacking, Mark explained their plan for managing the team, "Like our idea, divide it up into different sub parts and assign people different tasks from those. So that everybody has something to work on and can be utilizing their skills," (Mark Pre). In the end, Mark reflects and says "We tried our best to divide and conquer tasks, assign people where their strengths were so they were able to get tasks done quickly, and tried to stay on task," (Mark Post).

\section{Team $C$}

Devin became the leader in the group and explained his thoughts on the matter. "So I've heard, well I don't know where I read it, but there's how people become leaders. And they can either be appointed by people. They can either be just kind of, people will kind of accept that they're the leader because they have a lot of knowledge, or they know a lot, or they formed the group. Or everyone can decide who the leader is going to be. For this one it felt like it was based on what you know. So I pitched the idea. I have a lot of experience in it, in I guess the problem set and stuff," (Devin Pre).

\section{Discussion}

That data shows very interesting similarities and differences between hackathons and PBL courses. Almost all of the elements of the BIE Gold Standard PBL display themselves in some way through the hackathon environment. The whole structure of the hackathon is centered around hackers being able to choose projects for themselves without external influence such as an explicit educator. While this might immediately seem not PBL based, the structure of the hackathon itself acts as an educator to promote these PBL elements.

\section{A. Hackathon Project Selection and Hacker Interest}

The whole hackathon project process gives all decisions on the structure of the project to the team members. This directly shows the Student Voice and Choice element but also indirectly exhibits the Challenging Problem or Question element. The purpose behind these elements is to give students a vested interest in the project by making it their own. Because hackathon projects are thought up by the hackers themselves, their interest in its completion is present. Individuals in all teams indicated that the motivation they felt at hackathons to complete projects was much higher than that of their regular courses. Alex even claimed that there is "more at stake here" making them more invested in their projects (Alex Post). This attitude also shows the Authenticity of the process as hackers want to build something they want to build because they can.

\section{B. Skill Half Life}

One of the largest complaints that rapid-fire programming environments is the "short half-life" of the acquired skills [8]. Dr. Gary, a Project-Based Learning associate professor for Software Engineering at Arizona State University, criticizes "short-term, skills-focused learning activities" as they "do an inadequate job of placing those skills in context: a student might master skill X but is unable to assess tools and techniques that address the same problem space differently," [8]. However, Dr. Gary is primarily criticizing weekend programming courses, claiming that "Hackathons and Maker Faires are possible exceptions, as their relentless give-and-take over a short period can build unique communication skills," [8]. 
From the results, we see that hackers in a hackathon setting feel that the skills they've learned have a lasting effect on future projects. This is because, while hackathons may be a "short-term, skills-focused learning activity," it also has the elements of a PBL course. From the results, Mark explained that the way they learn things isn't necessarily optimal but it is a way to get it done as "a lot of time in software, there isn't necessarily a perfect or right way," (Mark Post). This study didn't do a follow up to see if the skills maintained their presence in the hacker's lives. Despite this, it is promising to say that some of the skills stuck with the hackers as many were very enthusiastic about the skills they learned and their future usage of it.

\section{Sustained Inquiry, Critique and Revision}

The hackathon setting does not have very explicit Sustained Inquiry, and Critique and Revision elements as the event happens at a pace that does not easily allow these. Many teams come to the hackathon with a few unrehearsed ideas that become the focus of their project. Typically, teams do research as they're developing which often leads to pivoting midway through the project, such as Team B. An interesting outlier is Team C's Devin who took it upon himself to do an abundance of research on land mine detection prior to the hackathon (Devin Pre). As stated in the results, some teams find ways to incorporate these elements by discussing with other teams or mentors but it is never an explicitly defined process.

The impact of these elements being poorly represented mean that the quality of product being created isn't necessarily the highest standard it could be. This is to be expected at a hackathon as a high-quality project, such as one from a semester long PBL course, is not feasible in a hackathon's small timeframe. This makes hackathon more focused on fast innovation instead of polished products. Mark explained that "you're not necessarily worried about how well it works, or if it's perfect, you just want to use it to some degree," (Mark Post). Even though the projects aren't of the highest quality, they are at a quality that make sense in the context of a hackathon. Also, the quality of the project doesn't necessarily reflect the quality of the learning they receive.

\section{Demoing and Success}

Hackathons have a very low bar in terms of success: being able to demo your product to the public. This aligns well with BIE Gold Standard PBL's element of Public Product as all hackers will be fully aware that their first goal is to display something to the public, which is a prerequisite to winning prizes. Unlike a final presentation and demonstration that you'd find in a PBL course, there isn't a grade attached to the quality of the final project in a hackathon ${ }^{1}$. Alex highlights that the Public Product itself provides enough motivation to hackers to perform well as there is a "social aspect" to being able to show the world what you've created (Alex Post).

\section{Conclusion and Future Work}

As shown through the study, hackathons have promise as a PBL environment and should be studied as such. Most every element of BIE's Gold Standard PBL make an explicit or implicit appearance in the hackathon setting. The lack of explicit Sustained Inquiry, and Critique and Revision might make hackathons something similar to PBL courses but with a much stronger focus on innovation rather than practicality. Regardless of the difference in structure, hackathons promote the same Key Learning, Understanding and Success skills that one would expect from a PBL course. Because of this, hackathons should continue to be analyzed in a more structured manner. An interesting research study might be developing a hackathon like course as a real 
university class. This class could help the engineering education community better understand the effect on learning that hackathons have. Other studies could generate hackathons with specific learning goals in mind and try to steer students into a curriculum while still maintaining the typical structure of a hackathon. This event would help show the worth of hackathons as an educational tool.

Another point of view for future works could be project management. The types of time and resource managements that participants exhibit within classroom and hackathon settings may be drastically different. These skills can be vital to a project and their appearance may differ with the differing environments. This work would focus more on the style of teamwork rather than the style of learning and is outside the scope of this paper.

A limitation of this study was how heavily focused the data collection was on individual teams. The observational protocol limited the data collection process to small numbers of teams per hackathon. In the future, a revised observational protocol that would allow for more teams to collect data from would be optimal. This change would provide more data to analyze which would give the study a broader range of individuals to review.

\section{References}

[1] S. Jordan and M. Lande, "Practicing Needs-Based, Human-Centered Design For Electrical Engineering Project Course Innovation," American Society for Engineering Education, p. 25.1041.1, 2012.

[2] J. Larmer and J. Mergendoller, "Gold Standard PBL: Essential Project Design Elements," Buck Institute for Education, 2015.

[3] Major League Hacking, "Major League Hacking," 2017. [Online]. Available: https://mlh.io. [Accessed 224 2017].

[4] G. Briscoe and C. Mulligan, "Digital Innovation: The Hackathon Phenomenon," Creativeworks London, 2014.

[5] J. Mills, "Engineering Education - Is Problem-Based or Project-Based Learning the Answer," Australian Journal of Engineering Education, 2003.

[6] J. Rice, "Hackathon Implementation for Industry and Academia," ProQuest, Ann Arbor, 2015.

[7] J. Mtsweni and H. Abdullah, "Stimulating and Maintaining students' interest in Computer Science using the Hackathon Model," Independent Journal of Teaching and Learning, vol. 2015, no. 10, pp. 85-87, 2015.

[8] K. Gary, "Project-Based Learning," The Institute of Electrical and Electronics Engineers Computer Society, vol. 48, no. 9, pp. 98-100, 2015 